\title{
Algunas características clínicas de pacientes con retardo de crecimiento
}

\author{
Dra. Isabel Torreaiba M. ${ }^{\text {; }}$ Dra. Matilde Maddaleno H. ${ }^{2}$; Dr. Francisco Beas F. 3 ; \\ Dra. Lilian Cuadra C. ${ }^{4}$; Dra. América Espinoza M. ${ }^{4}$; Dra, Amanda Cortínez R. ${ }^{3}$; \\ Dra. Martha Eggers M. ${ }^{3}$; Dra. Cecilia Hentíquez del V. 3
}

\section{Clinical aspects in patients with growth retardation}

\begin{abstract}
A retrospective study to evaluate the clinical characteristics of growth retardation (GR) was conducted through the analysis of 2.118 patients of the Children's Endoctinology Unit of the Paula Jaraquemada Hospital, corrcsponding to the last two years. $34 \%$ of these children ( 700 cases) presentes this disorder. Half of these patients were referred for shout stature; the most frequent causes were constitutional and;or familial $(42.7 \%)$. School childsen and adolescents complained most frequently. Growth retardation of genetic origin was more frecuent in low birth weight infants who were snall for gestational age (SCA). In GRs due to endocrine, genetic. or other causes, a higher percentage of psychomotor retardation and inadequate scholarity was found than in the constitutional and/or familial GRs. Stature and growth velocity was more compromised in genetic and endocrine than in constitutional and/or, familial GRs. Bone age showed greater retardation in endocrine GRs. Emphasis is placed upon the importance of an adequate clinical study, and through evaluation of the patients', pattern of growth, which is fundamental for early and accurate diagnosis

(Key words: Growth retardation, short statute, genetic, endocrine).
\end{abstract}

Para que un individuo exprese el maximo de su potencial biológico de crecimiento y desarro. llo, se requiere la interacción de numerosos factores: genéticos, nutricionales, hormonales y ambientales ${ }^{1,6}$. La talla es una medición de la longitud corporal y que evaluada en el tiempo en comparación con curvas de crecimiento normales, para la población estudiada, es muy útil en el estudio del crecimiento del niño. Hay una estre-

1. Becado en Endocrinología y Genética Infantil. Unidad de Endocrinología y Genética Infantil.

2. Becado en Adolescencia y A tención Primaria. Departamento de Pediatría, Hospital Luis Calyo Mackenna.

3. Instituto de Investigaciones Clínicas, División Ciencias Médicas Sur, Facultad de Medicina, Universidad de Chile. Hospital Paula Jaraquemada.

4. Becados Servicio de Pediatría. cha relación entre la normalidad del crecimiento y el estado de salud del individuo ${ }^{5,9}$. La percepción de la imagen corporal que los padres tienen del niño como la de este mismo al compararse con sus pares, es un importante motivo de consulta. El objetivo de este trabajo fue estudiar las causas del problema y algunas características clínicas de pacientes que consultaron por retardo de crecimiento a un servicio de endocrinologia y genética infantil durante un periodo de dos años.

\section{MATERIAL Y METODOS}

Se revisaron en forma retrospectiva 2.118 fichas de la unidad de endocrinología y genétjca infantil del Hospital Paula Jaraquemada entre los años 1982 y 1984: se encontraron 700 pacientes (34\%) con retardo de crecimiento, cuyos antecedentes sociodemográficos 
(sexo, edad, escolaridad), motivo de consulta, antecedentes perinatales, antecedentes mórbidos, del desarrollo psiconotor, ritmo de crecimiento, edad ósea y diagnóstico final fueron revisados para este análisis. Para la evaluación antropométrica se utilizaron las tablas de Tanner y col. ${ }^{\mathrm{B}} \mathrm{El}$ estudjo estadístico de las diferencias se evaluó mediante pruebas de Student y $\mathrm{Cl}$ cuadrado, con un rivel de significancia inferior a $5 \%(\mathrm{p}<0,05)$.

\section{Definiciones}

El retardo de crecimiento (RC) fue definido como talla inferior al percentil 3 según tabla de Tanner ${ }^{8}$. Se consideró de causa endocrina cuando ésta existía, turiese o no origen genético9,10. Se clasificó el RC como enfermedad genética en los casos con enfermedades mono o poligénicas, aberraciones cromosómicas y síndromes genéticos de mecanismo desconocido11. Se estimó que había un RC constitucional o familiar o ambos, (en adelante constincional o familiar) si el paciente tenía velocidad de crecimiento normal, talla baja, paralela al percentii 3 , edad ósea normal o levemente disminuida $\mathrm{y}$ antecedentes frecuentes de talla baja familiar 9,12

El ritmo de crecimiento medido como incremento de la talla, en centímetros, en un periodo no inferjor a 6 meses, fue considerado "regularmente leлto" en los pacjentes en que presentaban un ritmo hasta un $80 \% \mathrm{dcl}$ patrón de normalidad de Tanner y "muy lento" cuando era inferior a esta cifra.

E) déficit estatural (\% de la talla ideal para la edado p 50 de Tanner) fue calificada como severo si era igual o Inayor que $21 \%$ moderado entre 15 y $20 \%$ y leve entre 7 y $14 \%$ Los pacientes con déficit igual o menor que $6,9 \%$ están sobre el percentil $3 . y$ por definición no tienen $\mathrm{RC}$.

La escolaridad se calificó de acuerdo o no según la edad promedio aceptada para cada grado escolar por el Minjsterio de Educación.

\section{RESULTADOS}

Entre los 700 pacientes afectados por retardo de crecimiento, $51,3 \%$ eran mujeres $y$ un tercio tenian entre 10 y 13 años 11 meses de edad. En este grupo etario los varones consultan más frecuentemente. El motivo de referencia consignado en la ficha fue en $58,4 \%$ por retardo de crecimiento, en $18,6 \%$ por sospecha de enfermedad endocrina, en $18,7 \%$ por enfermedad genética y en $3,3 \%$ por otras causas. Los diagnósticos finales: $42,6 \%$ corresponden a RC constitucional o familiar, $26,7 \%$ a enfermedad genética, $20,3 \%$ a enfermedad endocrina y el $9 \%$ por RC debido a otras causas.

Entre los pacientes con RC constitucional o familiar, $58 \%$ eran hombres. La edad de consulta más frecuente en estos pacientes estaba entre 10 años y 13 ańos 11 meses; $70 \%$ de los hombres y $55 \%$ de las mujeres consultaron despues de los 10 años.

De los pacientes controlados por enfermedad endocrinológica con $\mathrm{RC}, 30 \%$ corresponden a hipotiroidismo, 25,5\% a raquitismo resistente y $15 \%$ a insuficiencia hipofisiaria.
Al analizar los antecedentes perinatales de los pacientes con $\mathrm{RC}$, se observó que $27 \%$ habían sido pequeños para la edad gestacional, y $24.7 \%$ tuvieron bajo peso de nacimiento. Los niños con $\mathrm{RC}$ por enfermedad genética presentaron con mayor frecuencia peso de nacimiento (PN) me. nor de $2.500 \mathrm{~g}(32,4 \%)$ y antecedente de PEG $(42 \%)$; estos porcentajes son significativamente mayores que en sujetos con $\mathrm{RC}$ constitucional o familiar y que en los RC de causa endocrina. El antecedente de retraso psicomotor se encontró en $53 \%$ de los RC por enfermedades genéticas, en $32 \%$ de los RC endocrinos, y en $34 \%$ de los RC de otras causas, pero sólo en $12 \%$ de los constitucionales o familiares. La escolaridad no adecuada se observó en un $59 \%$ de los RC endocrinos, $34 \%$ de los RC por enfermedad genética, $y$ $49 \%$ de los RC por otras causas, en cambio sólo ocurrió en $15 \%$ de los RC constitucionales o familiares.

El estado de nutrición evaluado por la relación peso/talla, mosttó desnutrición más frecuentemente en los pacientes genéticos $(22 \%)$ que en Ios constitucionales o familiares $(4 \%)$.

El déficit estatural se relacionó tambièn con la etiología del RC: los pacientes endocrinos y genéticos presentaron con mayor frecuencia déficit severo, mientras los niños con RC constitucional o familiar en su mayoría tenjan $\mathrm{RC}$ moderado o leve (Tabla I y Figura 1). E] ritmo de crecimiento estaba notoriamente más comprometido en los pacientes endocrinológicos, genéticos o por otras causas, que en aquellos de etiología constitucional o familiar. Cuando se analiza la edad ósea del total de los pacientes con RC, se observa que $60 \%$ de los pacientes con RC de causa endocrina, tenian severo compromiso de ella (más de 3 años de atraso). Por el contrario los pacientes con RC constitucional o familiar, los genéticos o por otras causas, en su mayoria $(80 \%)$ presentaban atrasos óseo leves o moderados (menos de 2 años).

En los pacientes constitucionales o farniliares, se estudiaron los antecedentes perinatales; relacionándolos con el ritmo de crecimiento, la edad ósea y el déficit estatural. Se observó que en este tipo de RC, el ritmo de crecimiento es independiente del peso de nacimiento (p no significativo) pero está relacionado con el antecedente de pequeño (PEG) o adecuado (AEG) para la edad gestacional: en 32 pacientes con antecedentes de PEG se registró con mayor frecuencia ritmos de crecimiento "muy lento" ( $65 \%$ ) en cambio de los 137 pacientes con antecedentes de AEG, el ritmo fue más satisfactorio en $71 \%(\mathrm{p}<0,05)$. Esta situación se repite al relacionar el atraso de la edad ósea, encontrándose que los pacientes con 

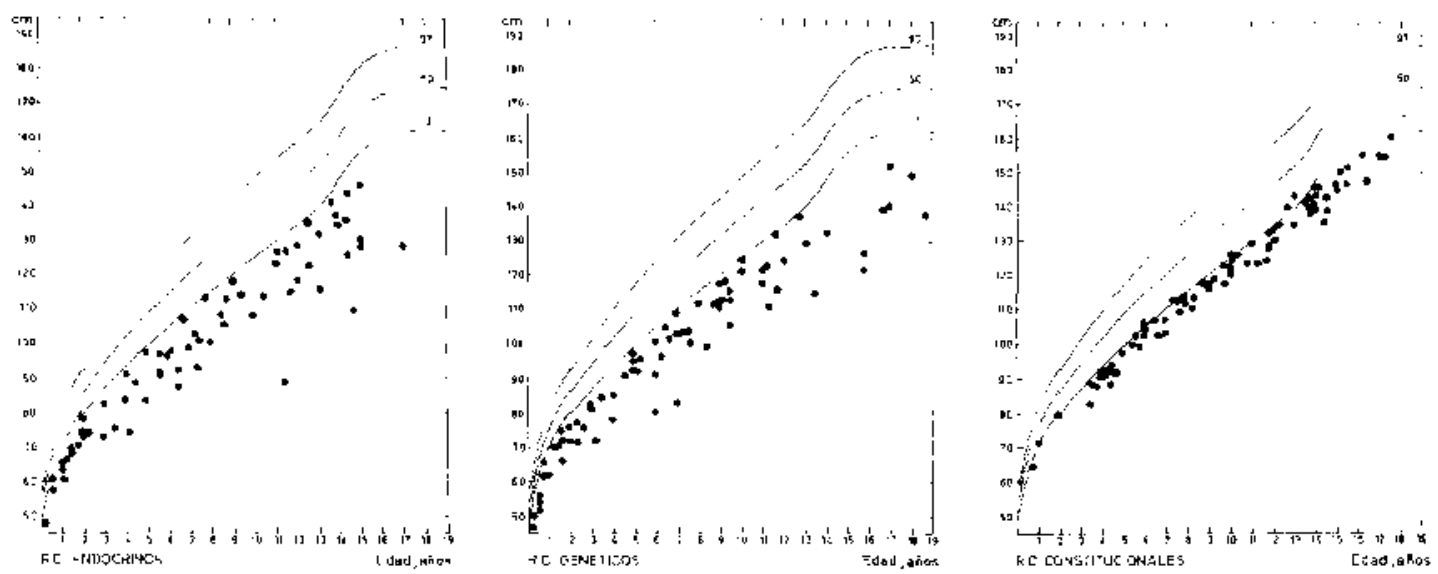

Figura 1. Talla de los pacientes con retardo de crecimiento (RC) endocrino (izqujerda), RC genético (centro) y RC constitucional (dereç3). Los pacientes con RC endocrino o genética presentan con mayor frecuencia lun déficit de talla severo. En los RC constitucional este retardo es leve o moderado.

Tabla I.

Déficit Estatural y causas etiológicas en paciontes con recardo de crecimiento

\begin{tabular}{|c|c|c|c|}
\hline $\begin{array}{l}\text { Déficit } \\
\text { Fistatura! }\end{array}$ & $\begin{array}{c}\text { Endocrinos } \\
\left(\begin{array}{c}\mathrm{n}=142) \\
\%\end{array}\right.\end{array}$ & $\begin{array}{c}\text { Cienéticos } \\
(\mathrm{n}=300) \\
\%\end{array}$ & $\begin{array}{c}\text { Constitu- } \\
\text { cionales } \\
\text { y/o } \\
\text { familiares } \\
(\mathrm{n}=187) \\
\%\end{array}$ \\
\hline $\begin{array}{l}\text { Severo } \\
(\geqslant 21 \% \text { déficit })\end{array}$ & $16,2^{*}$ & $11,0^{*}$ & $3,3^{*}$ \\
\hline $\begin{array}{l}\text { Moderado } \\
\{15-20 \% \text { deficit }\}\end{array}$ & 20,0 & 26,0 & 23,0 \\
\hline $\begin{array}{l}\text { Leve } \\
\text { ( } 714 \% \text { déf jeit) }\end{array}$ & 66,0 & 63,0 & 73,0 \\
\hline
\end{tabular}

RC constitucional o familiar y antecedente de PEG, presentaban más frecuentemente atraso óseo de más de 2 años que los pacientes AEG. Lo mismo sucede con el déficit estatural, encontrándose más comprometida la talla en pacientes con antecedentes de PEG que en los AEG.

Al comparar la edad ósea con el ritmo de crecimiento de los pacientes con RC constitucional o familiar, se observa que en los pacientes con menos de 2 años de atraso óseo $(n=121)$, el ritmo de crecimiento es "regularmente lento" en $86 \%$ y sólo en $14 \%$ es "muy lento"; en cambio cuando el atraso óseo es mayor de 2 años $(\mathrm{Il}=67)$, el ritmo de crecimiento es más frecuentemente, muy lento ( $54 \%$ ). Algo similar ocurre al relacionar el atraso óseo con el déficit estatural, en estos pacientes con RC constitucional o fami- liar: mientras mayor es el atraso óseo mayor es el déficit estatural como era de esperar. En la Tabla 2 se resumen las características generales de los pacientes con RC.

\section{DISCUSION}

Treinta y cuatro por ciento de los pacientes atendidos en esta unidad de endocrinología infantil y genética, presentan retardo de crecimiento. La mitad consultó por esta causa y la etiolo. gía más frecuente es la constitucional o familiar $(42,7 \%)$. Los pacientes consultan más frecuentemente en la edad escolar y la adolescencja.

La mayoria de las afecciones que causan RC pueder ser diagnosticadas clínicamente. Para lograr este objerivo es fundamental conocer los antecedentes perinatales, problentas nutricionales, antecedentes familiares, enfermedades crónicas, realizar un completo examen físico y tratar de identificar un "canal de crecimiento" que permita reconocer la edad de inicio del retraso y la velocidad de crecimiento en el mo. mento del control ${ }^{9,14}$. En este estudio se corroboran algunos hechos descritos en la literatura. Los $\mathrm{RC}$ por enfermedades genéticas tienen más frecuentemente PN menores de $2.500 \mathrm{~g}$ y antecedentes de PEG ${ }^{15}, 16$; los RC de etiología endocrina, genética y por otras causas, mayor frecuencia de retardo psicomotor $y$ escolaridad atrasada que los RC constitucional o familiar. En los RC endocrinos y genéticos el compromiso de la talla es mayor que en los RC constitucionales o familiares. El compromiso acentuado de la edad ósea es característico de los RC de causa endocrina $^{9}$. La velocidad de crecimiento en los pacientes constitucionales o familiares es mejor que 
en los casos endocrinos y genéticos. A medida que el déficit estatural es mayor y la velocidad de crecimiento menor, aumenta la probabilidad de que el RC sea de causa endocrina, genética o por enfermedad sistémica $9,10,13$.

La importancia del crecimiento intrauterino adecuado para el normal desarrollo posterior se evidencia en los pacientes con antecedentes de ser pequefíos para la edad gestacional. En la literatura se describe que los pacientes con retardo de crecimiento con antecedentes de PEG, $\sin$ otra enfermedad, representan 6 a $17 \%$ de todos los casos con retardos de crecimiento 19,20 y que ellos siguen siendo pequeños, con ritmos de crecimiento más lentos y edades ósea más atrasadas que los pacientes $A E G^{15.1 B}$. No está clara la causa de este retardo de crecimiento y se supone una alteración intrínseca en la multiplicación celular durante la vida intrauterina, con posterior disminución del potencial de crecimiento ${ }^{1.3}$. En este estudio los pacientes con RC constitucional o familiar tenían en $20 \%$ antecedentes de PEG, mayor compromiso estatural, ritmo de crecimiento más lento y edad ósea más atrasada que los niños $\mathrm{AEG}$.

$\mathrm{La}$ edad ósea sigue siendo un elemento clínico que aporta una orientación diagnóstica y es útjl en el pronóstico de los pacientes con talla ba$\mathrm{ja}^{9,13,14}$. Nuestros pacientes con RC constitucional o familiar, tenian edades óseas iguales a la edad estatural, hecho que los diferencia de los
$\mathrm{RC}$ endocrinos, que, en general, tienen edades óseas más atrasadas que la edad estatural ${ }^{13,14}$.

El retardo de crecimiento es uno de los aspectos que más preocupan a los padres, especialmente en el hijo varón. La importancia que el niño asigna a-su estatura, preferentemente en el período puberal, tiene impacto psicológico importante, tanto en su auto imagen como en el desarrollo de la personalidad, lo que hace necesario el diagnóstico precoz y el tratamiento adecuado de este problema 21,22 . Según nuestro conocimiento, éste es el primer trabajo en nuestro país que analiza la frecuencia y características clínicas generales de niños con retardo de crecimiento que consultan en un policlínico de endocrinología. En general esas características son similares a las publicadas por otros autores: Horner en 263 ninos con RC encontró $57,6 \%$ de RC constitucional, $17 \%$ por enfermedad sistémica, $10 \%$ de origen endocrino, un $9 \%$ debido a enfermedad genética, y un $6,5 \%$ secundario a retardo intrauterino 21 . En una serie de 429 pacientes descrita en Argentina, $292(68 \%)$ tenían RC constitucionales o familiares. Entre 137 enfermos, $18 \%$ tenían afecciones genéticas, $24 \%$ endocrinas, en $28 \%$ el RC tenía causas sistémicas y en $17 \%$ habian antecedentes de $P^{2} G^{22}$. Es importante recalcar que por tratarse de pacientes referidos a consultas especializadas estos estudios no reflejan la prevalencia de las afecciones ana. lizadas.

Tabla 2 .

Características clinicas generales en pacientes con retardo de crecimiento de causa constitucional y/o familiar, endocrina y genética

\begin{tabular}{|c|c|c|c|}
\hline & $\begin{array}{l}\text { Constitucional } \\
\text { y/o familiar }\end{array}$ & Endocrino & Genético \\
\hline Antecedentes gestacionales & AEG & $A E G$ & PEG \\
\hline Peso de nacimiento & $\begin{array}{l}\text { normal o } \\
\geqslant 2.500 \mathrm{gr}\end{array}$ & $\begin{array}{l}\text { notmal o } \\
\geqslant 2.500 \mathrm{gr} .\end{array}$ & $<2.500 \mathrm{gr}$ \\
\hline $\begin{array}{l}\text { Antecedentes mórbidos } \\
\text { o sistémicos }\end{array}$ & ausente & ausente & frecuente \\
\hline Escolaridad & normal & inadecuada & inadecuada \\
\hline Desarrollo psicomotor & normal & comprometido & comprometido \\
\hline Ritmo de crecimiento & $\begin{array}{l}\text { normmal } \\
\text { o lento }\end{array}$ & muy lento & muy lento \\
\hline Déficit estatural & leve & $\begin{array}{l}\text { modersdo } \\
\text { o severo }\end{array}$ & $\begin{array}{l}\text { moderado } \\
\text { o severo }\end{array}$ \\
\hline $\begin{array}{l}\text { Antecedente familiar } \\
\text { talla baja }\end{array}$ & presentes & raro & posible \\
\hline Edad ósea & $\begin{array}{l}\text { moderadamente } \\
\text { comprometida }\end{array}$ & $\begin{array}{l}\text { muy } \\
\text { comprometida }\end{array}$ & $\begin{array}{l}\text { moderadamente } \\
\text { comprometida }\end{array}$ \\
\hline
\end{tabular}




\section{RESUMEN}

Se revisaron 2.118 fichas de la Unidad de Endocrinología y Genética Infantil del Hospital Paula Jaraquemada, durante dos años; y se estudiaron en forma retrospectiva los retardos de crecimiento (RC), que corresponden a $34 \%$ (700 casos). La mitad de ellos consultó por esta causa, siendo su etiología más frecuente la constitucional o familiar $(42,7 \%)$. Las edades de consulta más frecuentes fueron las escolares y la adolescencia. Los RC por enfermedad genética tienen más frecuentemente bajo peso al nacer y antecedentes de PEG. En los RC por enfermedad endocrina $_{i}$ genética o por otras causas, se observa mayor proporción de pacientes con retraso psicomotor y escolaridad inadecuada que en los $\mathrm{RC}$ constitucionales o familiares. La talla y velo. cidad de crecimiento está más comprometida en los RC debidos a enfermedad genética $y$ endocrina que en los constitucionales o familiares; y la edad ósea está más retrasada en los RC endocrinos que en el resto de los RC. El adecuado estudio clínico y la identificación del canal de crecimiento de estos pacientes, son elementos fundamentales para hacer oportunamente el diagnóstico.

\section{AGRADECIMIENTOS}

Nuestros agradecimientos especiales a los señores Daniel Alvo y Getmán Larraín por su valiosa colaboración en el estud io estadístico.

\section{REFERENCIAS}

1. Beas $F$. F Endocrinología del Crecimiento y DesarroLlo; Cap. 172 Mencghello, J. Pediattia, 3a. Ldición, Editorial Publicaciones Técnicas Mediterráneo Ltda. Santiago. 1985. pp. 1174-1179.

2. Rimoin D.L., Horton W.A.: Short Statuse. J. Pediatr, 92: 523 y 697, 1978.

3. Frasier D.: Trastornos del Crecimjento en niños: Clin. Pediatr. N.A. 26: 3, 1979.

4. Shepard $T$. .: Normal and abnomal growth patterns: in 2nd Edition, Gardner L. Endochrine and genetic disease of children and adolescense. Saunders W.B. Co. Philadelphia, 1975. pp. 11-99.
5. V'aughn I'.C.: en Nelson, W, Pediatria: 7a, Fdicion. Ed, Salvat S.A., Barcelona, 1980. pp . 13-46.

6. Garcia $H_{\text {: }}$ Retraso de erecimiento. Winter A.: Manual de Pediatría. Ed. I.M.C. Color Ltda. Santiago, 1984, pp. 486-509.

7. Patri A. y col.: Crecimiento y Desarrollo. Salud escolar. Ed. A. Bello, Santiago, 1979. pp. 83-103.

8. Tanner J.M., Whitehouse R.H., Takahishi M.: Standards from birth to madurity for height, weight, height velocity, weight yelocity in British children. Arch. Dis. Child. 41; 454 y 615, 1966.

9. Nissley P.: Endocrine causes of short stature U.S.A. Fndrocrinology section; Metabolism branch of Nat Jonal Cancer Institu te. NH, 1985, pp, 40.

10. Rudman D., Kutner $M$., Blackstown D., Janssen R., Patterson J.: Norma] variant of short stature, subclassification based on responses to exogenous growth hormone treatment, J. Clin, Endocrinol. Metab. 49: 92, 1979 .

11. Smith D.: Atlas de malformaciones somáticas en el niño. Ed. Pediátrica, 1ra. Edición española. Batcclona, 1972 , pp. $1-32$.

12. Beas y col: Retardo de crecimiento en el nirio. Norma de enfermedades endocrinológicas en pediatría. Unidad de endocrinología y genética infantil, servicio de pediatría, hospital Paula Jaraquemada: 1978. pp. 36.

13. Van wyke J.: Diagnosis and treatment of growth Failure. Hosp. Pract. 13: 145, 1984.

14. Schoff Blass $E_{.+}$Burtein $S_{1}$, Rosenfield $R_{.}$: Advances and treatment of short stature, with special reference to rol of growth hormone: J. Pediatr. 104: $801,1984$.

15. Cruise $M$.: A longitudinal study of grow th of low birth weight infants. Pediatrics. S1: 620, 1973.

16. Ounsted M., Taylor M.E.: The postnatal grewth of children who were small for date at birth. Develop. Med. Child. Neurol. 13: 421, 1971.

17. Hack M.: The prognostic significance of postnatal growth. in very low birth weight infants. Am. $J$. Obstet. Gynecol. 143: 693, 1982 .

18. Davies D.P.: Nutritional status of low birth weight infant and its influence on early postnatal growth. Arch. Dis. Child. 54: 703, 1979

19. Allan and Root J.: Diagnóstico y manejo del retardo de crecimiento en el niño. J. Pediatr. 78: 707,1971 .

20. Jacey and Parkin J.: Short Stature. Arch. Dis. Child. 49: 417, 1974.

21. Hornet J.M., Thorson A. Raymond L.: Growth deceleration patterns in children with constitutional short stature. Pediatrics 62: 529, 1978.

22. Martinez A., Cianis L, Heinrich J.J., Rodríguez L., Bergedó $C$.: Evaluation of short stature in children. Helv. Paediatr. Acta 37: 563, 1982. 